\title{
Cellular automata on high-dimensional hypercubes
}

\author{
Leonardo G. Brunnet ${ }^{1}$ and Hugues Chaté ${ }^{2}$ \\ ${ }^{1}$ Instituto de Física, Universidade Federal do Rio Grande do Sul, Porto Alegre, Brazil \\ ${ }^{2}$ CEA-Service de Physique de l'État Condensé, CEN Saclay, 91191 Gif-sur-Yvette, France
}

(Received 7 January 2004; published 18 May 2004)

\begin{abstract}
The emergence of nontrivial collective behavior is studied in large families of cellular automata rules implemented on high-dimensional hypercubes. Evidence is found that the region of rule space where such macroscopic dynamics exists is well-defined in the infinite-dimension limit.
\end{abstract}

DOI: 10.1103/PhysRevE.69.057201

PACS number(s): 05.45.Xt, 05.65.+b

\section{INTRODUCTION}

The modeling of a variety of problems in physics, chemistry, or even biology may be approached by using cellular automata (CA) [1-3]. Since the beginning of the last decade [4], it has been known that such systems, constructed with simple local rules and a small number of local states, may present a chaotic and disordered local behavior together with a well-defined, low-dimensional, temporal evolution of (spatial) averages. Termed "nontrivial collective behavior" (NTCB), these macroscopic attractors usually have a basin of finite measure, and their number (in phase space) is independent of system size. In a mean-field approach, one could expect that the variety of global attractors for the dynamics would decrease at high dimensions, since in that case the neighborhood of each site can be thought to be a good representative of the average behavior of the system. However, previous results exploring up to six dimensions show the opposite: the diversity of attractors increases. [5,6]

With the exception of Refs. [5,6], high-dimensional cellular automata have not been explored much, mainly because they require large computational resources. In this work, we focus on exploring these prototypical systems in even higher dimensions. Using a convenient numerical algorithm, it is possible to implement the dynamics of a cellular automaton on a hypercube and reach up to 25 dimensions, albeit, naturally, with a minimal linear extent. In order to identify the collective behavior of our CA rules, we monitor the evolution of the simplest global observable. Particular attention is paid to the evolution, as space dimension increases, of the region of rule space where bona fide NTCB is found, in an attempt to approach the infinite-dimension limit.

\section{CA RULES ON THE HYPERCUBE}

We consider a collection of $2^{\mathcal{D}}$ discrete variables $x$ $\in\{0,1\}$ sitting at the vertices of a $\mathcal{D}$-dimensional hypercube. The initial condition is constructed by randomly distributing $x=1$ values on the hypercube with a concentration $c_{0}$, the remaining sites being set to $x=0$. The dynamics is implemented in parallel synchronous time steps by applying the following deterministic cellular automata rule depending only on $S$, the sum of the values of the $\mathcal{D}$ nearest neighbors of a given site (the so-called "totalistic" rule):

$$
x^{t+1}= \begin{cases}1 & \text { if } S_{\min } \leqslant S^{t} \leqslant S_{\max } \\ 0 & \text { otherwise }\end{cases}
$$

Limiting ourselves to all combinations where $0<S_{\min }<S_{\max }<\mathcal{D}$ defines Game of Life-like rules ( $S_{\min }>0$ and $S_{\max }<\mathcal{D}$ ensures that the homogeneous $x=0$ state is the only absorbing state). These rules, denoted by $\mathcal{R}_{S_{\text {min }}, S_{\text {max }}}^{\mathcal{D}}$, form a family of reasonable size. The number $\mathcal{N}_{\mathcal{D}}$ of possible rules for a $\mathcal{D}$-dimensional hypercube is

$$
\mathcal{N}_{\mathcal{D}}=\sum_{i=1}^{\mathcal{D}-1} i=\frac{(\mathcal{D}-1)(\mathcal{D}-2)}{2} .
$$

Numerically, the simulation of such CA rules is facilitated by the use of a bit string representation for the vertices of the $\mathcal{D}$-dimensional hypercube. Each site can be encoded by a $\mathcal{D}$-bit string, where each bit codes the coordinate ( 0 or 1$)$ along one dimension. The bit string associated with a neighbor of a given site then differs from the bit string associated with that site by one single bit. The first neighbors of a given vertex may thus be found by an XOR (exclusive OR) operation between the bit string associated with that vertex and the bit string associated with $2^{i}, i=0, \ldots, \mathcal{D}-1$. Figure 1 illustrates the $\mathcal{D}=3$ case. These easily programed operations are rather fast on a computer.

\section{RESULTS}

For convenience, we mostly monitored $c(t)$, the average value of the sites (or, equivalently, the density of $x=1$ sites)

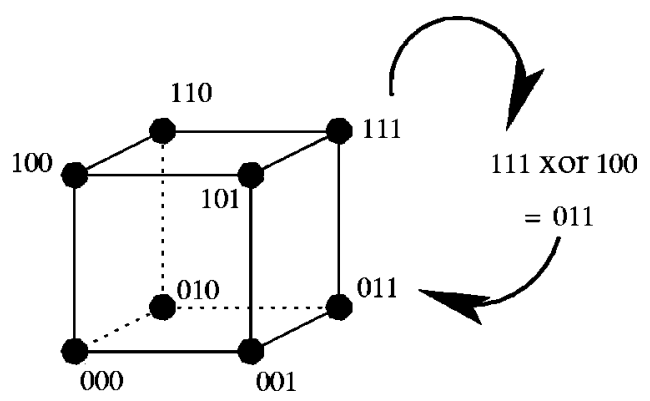

FIG. 1. Bit string representation of the vertices of a hypercube, $\mathcal{D}=3$, and the corresponding bit operation to find the nearest neighbors. 


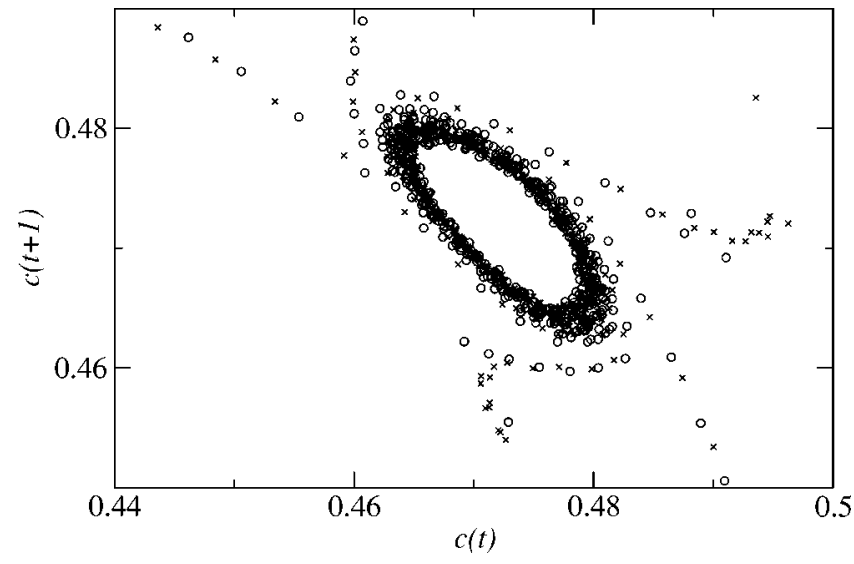

FIG. 2. First return map of a typical collective quasiperiodic QP5 regime (irrational period close to 5). Rule $\mathcal{R}_{8,12}^{25}$. The symbols indicate the different initial concentrations: circles for $c_{0}=0.25$ and crosses for $c_{0}=0.5$. The transients leading to the central torus are shown.

during the simulations. Both the concentration return map $[c(t+1) \times c(t)]$ and its time series $[c(t) \times t]$ are used to globally characterize the state of the system. For each possible rule $\mathcal{R}$, at least three different initial concentrations are used: $c_{0}=0.25,0.5,0.75$. This helps to ensure whether the collective motion recorded is a true attractor (i.e., with a large, finite-measure basin of attraction) or a "marginal" one (see below). We thus scanned the families of rules defined above in dimensions $\mathcal{D}=17-25$. We first describe the typical cases observed, characterize them, and define our notation.

The not-so-interesting situations, in the present context, are those where the asymptotic evolution is not chaotic. In the simplest case, all activity dies and every initial condition eventually leads to the $c=0$ absorbing state. These rules are coded $\mathrm{Z}$ in the following tables. It may also be that some rules take the system to locally periodic solutions (LP) where some specific sites follow an exact periodic cycle with a site-dependent period [5]. Apart from this remaining localized activity, the asymptotic state is then essentially frozen.

In agreement with previous findings [5], the true NTCB attractors fall into two basic classes: periodic $(\mathrm{P})$ or quasiperiodic (QP). Note that, strictly speaking, both are periodic but that QP rules have an irrational period, incommensurate with the clock of the discrete-time system. The macroscopic behavior is actually noisy, but with normal, statistical fluctuations which disappear in the infinite-size limit. These attractors are robust to perturbations such as some degree of noise in the rule, and they do not present obvious local order or structure in space. Figure 2 shows the collective quasiperiodic behavior with a period close to 5 (QP5) exhibited by the rule $\mathcal{R}_{8,12}^{25}$.

Attractors with a more complex structure may also exist, and they are generally found to be combinations of the above two cases. For example, we have found evolutions presenting a P2 basic cycle embellished with a QP3: the return maps at every other time step show a QP3 attractor. This has been denoted by $\mathrm{P} 2 \times \mathrm{QP} 3$ in our classification. Similarly, we have found $\mathrm{P} 2 \times \mathrm{QP} 3, \mathrm{P} 3 \times \mathrm{QP} 3$, etc.

An additional possibility is the occurrence of intermittent switching between two (or more) well-defined macroscopic

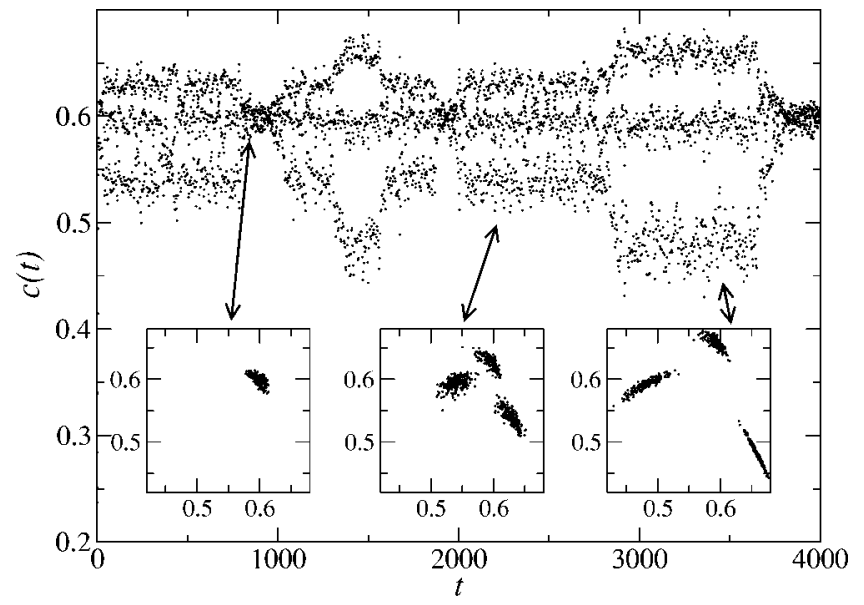

FIG. 3. Time series of $c$ for intermittent rule $\mathcal{R}_{6,11}^{17}$. Intermittent behavior between three collective regimes (two different P3, and one P1), as shown in the short-time return maps shown in the insets.

dynamics. A typical case is shown in Fig. 3. In the following the symbol "|" is used to indicate intermittent behavior among attractors (e.g., "P $\mid \mathrm{QP}$ " represents intermittent behavior between periodic and quasiperiodic attractors).

Cases where different initial conditions lead to different macroscopic dynamics are the most difficult to diagnose. In the large systems studied here, it is indeed quite impossible to distinguish between the coexistence of a few bona fide NTCB attractors, each with a finite-measure basin of attraction, and an infinity of different macroscopic dynamics with essentially zero-measure basins. Most of the time, the latter possibility seems to be the right one: any initial condition leads to a different, often complicated (i.e., not classifiable as $\mathrm{P}$ or QP) asymptotic dynamics. Activity is then often confined to parts of the system. These rules have been termed "marginal," coded M. Figure 4 shows first return maps for a typical marginal rule (for clarity the first 100 points were dropped).

With this taxonomy in mind we classified all possible rules. The results are shown in the $S_{\min } / \mathcal{D} \times S_{\max } / \mathcal{D}$ plane, which allows for a unified representation of our global re-

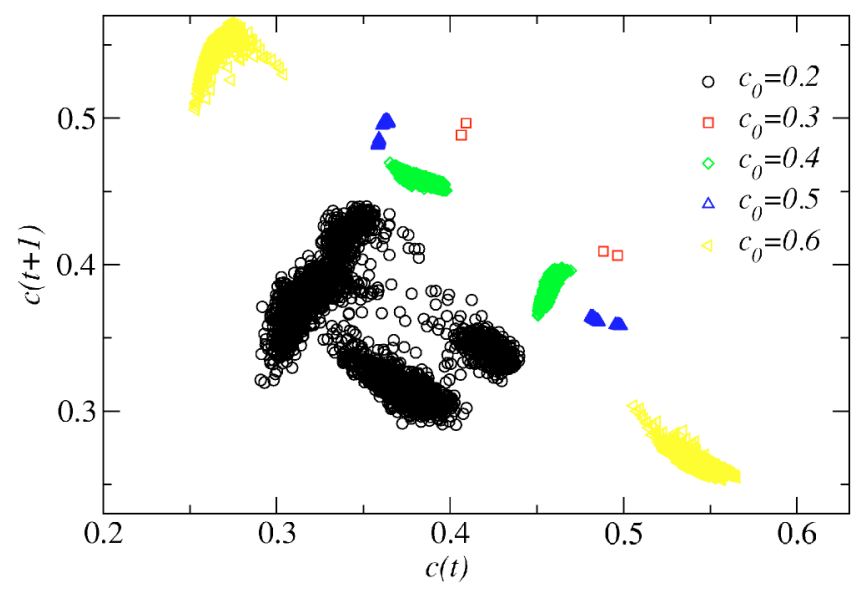

FIG. 4. First return map of a typical marginal rule $\mathcal{R}_{1,3}^{17}$. The symbols (and color online) indicate the different initial concentrations from $c_{0}=0.2$ to $c_{0}=0.6$. 

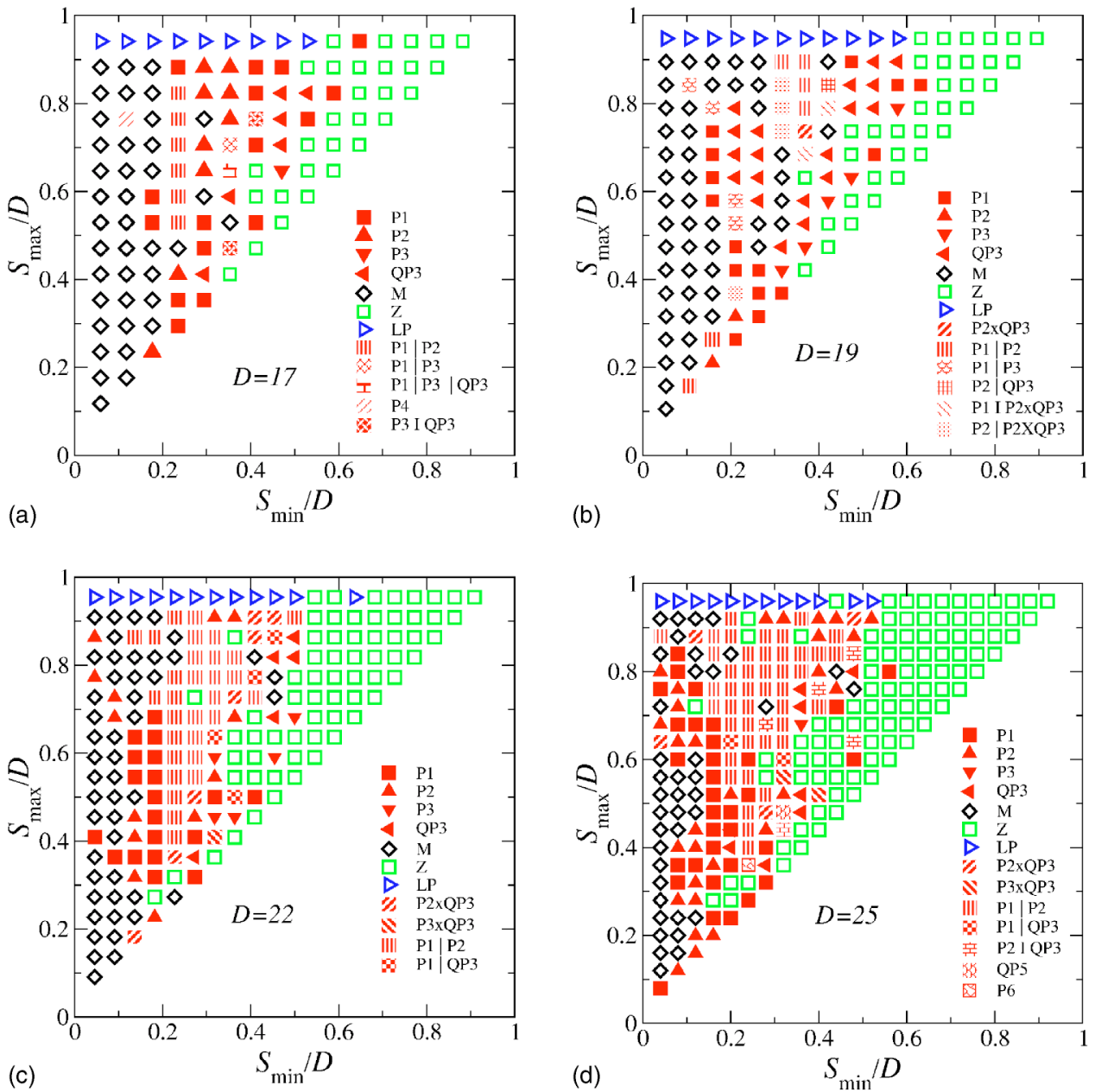

FIG. 5. (Color online) Classification of the dynamics for all possible rules on the hypercube for $\mathcal{D}=17,19,22,25$.

sults in all dimensions. To facilitate a global view of the partitioning of rule space, we used empty symbols (square, diamond, and triangle) to denote nonchaotic (Z, LP) or marginal (M) behavior. Solid symbols were reserved to represent NTCB. In Fig. 5, which shows rule space for dimensions $\mathcal{D}=17,19,22$, and 25 , one can thus clearly distinguish a large central region where NTCB is found, whereas marginal (M) behavior is confined to the left (small $S_{\min }$ ). Nonchaotic rules $\mathrm{Z}$ and LP are, respectively, found in the upper right (large $\left.S_{\min }\right)$ and upper left $\left(S_{\max } \sim \mathcal{D}\right)$ regions, in agreement with simple mean-field arguments [5].

\section{DISCUSSION}

Our results are of course limited, due to their numerical nature. Having deliberately chosen to go to very large dimensions, we could not apply systematically the necessary protocol to check whether our findings subsist in the infinite-size limit. We nevertheless suspect that they do so. At any rate, satisfactory answers will only come after one is able to predict from the local rule, within some (mean-field-like) approximation, the observed collective motion.

This caveat aside, several messages can be extracted from our results. One such conclusion, already hinted at in Ref.
[5], is that the mean-field approximation [or at least its simple implementation in terms of a single map for $c(t)$ ] does not represent the infinite-dimension limit of the behavior of chaotic, high-dimensional CA rules. More precisely,

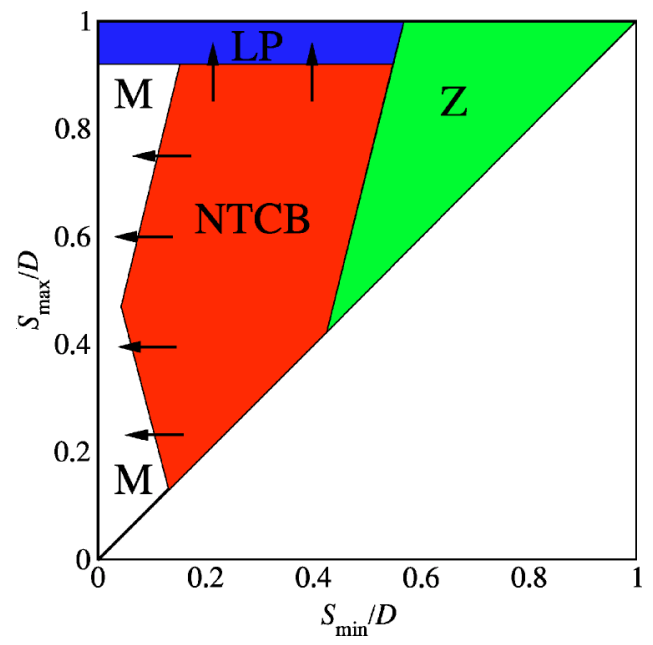

FIG. 6. Sketch of rule space in the infinite dimension limit. The arrows indicate the possible shrinking of $\mathrm{M}$ and LP regions in this limit (see the discussion in the text). 
the infinite-dimension limit seems to be singular, since extrapolating the results presented here to $\mathcal{D} \rightarrow \infty$ hints at a well-defined partition of rule space.

Figure 6 is an attempt at such an extrapolation to the infinite-dimension limit. The central NTCB region, as observed in Fig. 5, is well-defined and shows no sign of shrinking when increasing $\mathcal{D}$. One can observe, though, that intermittent behavior seems to occupy a larger and larger region of parameter space. This is due to the increasing diversity of NTCB combined with the increasing importance of finitesize effects in our size- 2 dimension- $\mathcal{D}$ hypercubes. We expect, as noted in Ref. [5], that intermittent behavior progressively disappears, at fixed $\mathcal{D}$, as the linear extent of the hypercube is increased, because it does not seem generic for many rules to sit precisely at the border between two basins of attraction. The status of marginal rules is also confirmed by our results: they do appear as sitting at the "edge of chaos" $[1,7]$ and are thus probably the most "complex" ones. Going one step further in our extrapolation, one can notice, from Fig. 5, that the marginal and locally periodic rules (M and LP) tend to occupy a smaller and smaller region as $\mathcal{D}$ increases. On the other hand, no such trend is apparent for zero (Z) rules. Accepting these observations would lead to an asymptotic picture slightly different from Fig. 6, where only NTCB and zero rules occupy a finite portion of rule space (the arrows in Fig. 6 are an attempt at indicating this possibility).

In a sense, the asymptotic existence of NTCB on the hypercube found here strengthens both the trend uncovered in Ref. [5] for rather large lattices of moderate dimension, and the findings of Mousseau [8], who showed that the NTCB is also generically observed in random networks of finite, constant connectivity (which may be thought of as being infinite dimensional). This suggests looking for NTCB in other geometries such as complex networks, with the additional difficulty that a different rule would then have to be implemented at different nodes because of the inherent varying connectivity of these systems.

\section{ACKNOWLEDGMENTS}

We thank Capes (Brazil) and Cofecub (France) for support. L.B. also thanks the CEA/Saclay for hospitality.
[1] S. Kauffman, The Origins of Order: Self-Organisation and Selection in Evolution (Oxford University Press, New York, 1993).

[2] S. Wolfram, Theory and Application of Cellular Automata (World Scientific, Singapore, 1986).

[3] N. Boccara, Modeling Complex Systems (Springer, New York, 2003).
[4] H. Chaté and P. Manneville, Europhys. Lett. 14, 409 (1991).

[5] H. Chaté and P. Manneville, Prog. Theor. Phys. Suppl. 87, 1 (1992).

[6] P. Binder, J. Stat. Phys. 68, 4 (1992).

[7] R. M. Zorzenon dos Santos and A T. Bernardes, J. Theor. Biol. 186, 173 (1997).

[8] N. Mousseau, Europhys. Lett. 33, 509 (1996). 